\title{
Different strategies towards the deterministic coupling of a Single QD to a Photonic Crystal Cavity Mode
}

\author{
J.Herranz ${ }^{1, *}$, I.Prieto ${ }^{1}$, Y.González ${ }^{1}$, J.Canet-Ferrer ${ }^{2}$, P.A.Postigo ${ }^{1}$, B.Alén ${ }^{1}$, L.González ${ }^{1}$, \\ L.J.Martínez ${ }^{1}$, M.Kaldirim ${ }^{1}$, D. Fuster ${ }^{2}$, G.Muñoz-Matutano ${ }^{2}$, and J.Martínez-Pastor ${ }^{2}$ \\ ${ }^{1}$ IMM-Instituto de Microelectrónica de Madrid (CNM-CSIC), Isaac Newton 8, PTM, E-28760 Tres Cantos, \\ Madrid, Spain \\ ${ }^{2}$ UMDO (Unidad Asociada al CSIC-IMM), Instituto de Ciencia de Materiales, Universidad de Valencia, P.O. \\ Box 22085, 4607 Valencia, Spain
}

A single Quantum Dot (QD) coupled to a photonic cavity mode is the fundamental system for the study of Cavity Quantum Electrodynamics (CQED) phenomena in the solid state approximation [1], a very promising field for the development of quantum information technologies. Strong requirements on the simultaneous spectral and spatial matching of the emitter and the optical cavity mode make the fabrication of single QD-cavity mode coupled system very challenging. Although coupling of single self-assembled QD to a photonic crystal (PC) cavity mode has already been demonstrated [2, 3], the technology is far from being mature. In this sense, the use of high spatial resolution lithographic techniques for site controlled QD formation $[4,5]$ is crucial in order to improve the yield of deterministic integration of a coupled QD - cavity mode[6,7].

In this work we present two strategies for coupling InAs site-controlled QD with the optical mode of GaAs-based PC microcavities. Site controlled InAs QDs are obtained using AFM local oxidation lithography to define preferential nucleation sites during MBE growth. These nanostructures show good optical emission and can be operated as single photon sources [5].

The first approach consists of the fabrication of PC microcavities on GaAs slabs. On top of the microcavities, AFM local oxidation lithography is performed to define the nucleation site of a single QD within the cavity. They are transferred into the MBE chamber to complete the structure to the target thickness with an embedded InAs QD at the predefined site. Our results show that this process leads to a strong evolution of the round shape of the PC holes, that degrades the quality factor $(\mathrm{Q})$ of the cavity mode.

In the second approach, same techniques are employed; an etched ruler is fabricated by using $e$-beam lithography and dry etching (RIE) on a GaAs epitaxial slab. Then, AFM local oxidation lithography is performed to define the nucleation sites of InAs QDs. The position coordinates are set and recorded with respect to the fabricated ruler. The sample is then completed by a MBE regrowth process. Once it is completed, PC cavity can be designed for matching the emission wavelength of the embedded QD and later fabricate around the previously recorded position coordinates of the QD with respect to the ruler. Optical microPL measurements carried out by confocal microscopy at $77 \mathrm{~K}$ of the obtained nanostructures in both approaches will be shown.

[1] T. Yoshie et al., Nature 432 (2004) 200.

[2] A. Badolato et al., Science, 308 (2005), 1158.

[3] K. Hennesy et al., Nature 445 (2007), 896.

[4] T. Sünner et al., Opt. Lett 33 (2008), 1759.

[5] J. Martin-Sanchez et al., ACS Nano 3 (2009), 1513.

[6] S. M. Thon et al., App Phys Lett 94 (2009) 111115.

[7] D. Englund et al., Proc. SPIE 7611 (2010) 7611 OP.

*Contact: jesus@imm.cnm.csic.es 


\section{Figures}

a

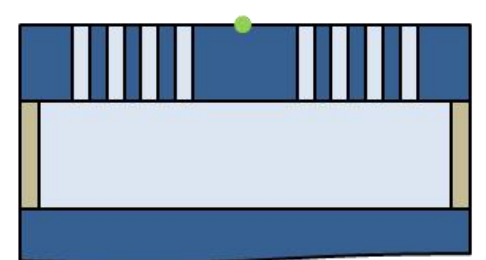

Oxide point

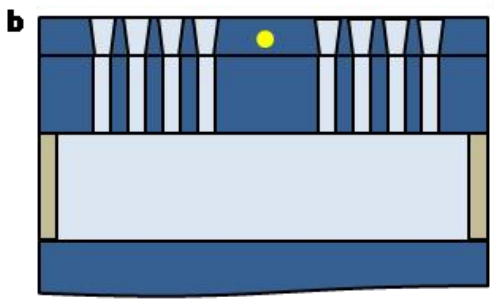

InAsQD
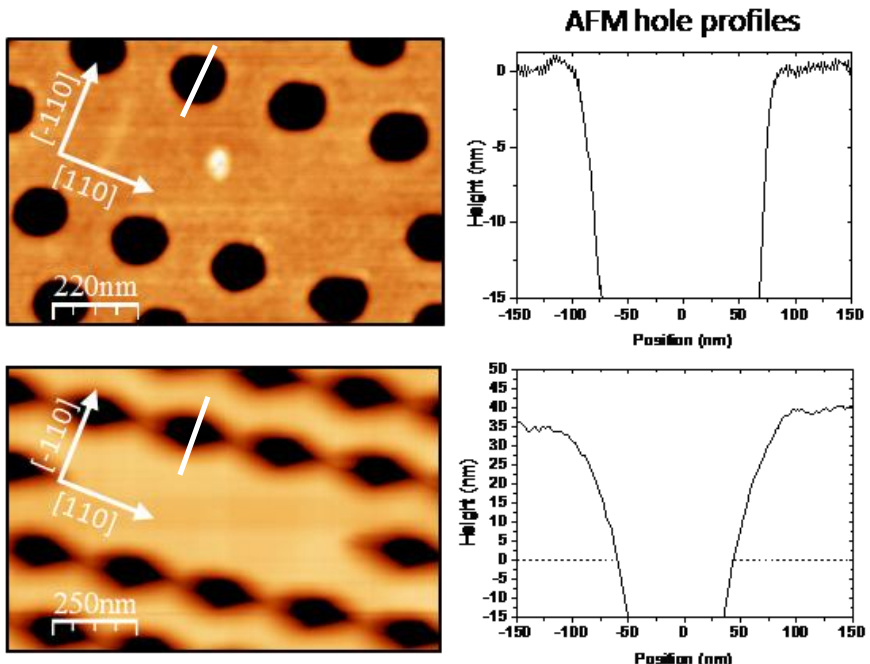

Figure 1. First approximation: Site controlled InAs QD fabrication at the maximum of the electric field of a pre-patterned photonic crystal cavity. Different process steps and the corresponding AFM images: (a) Fabrication of the photonic crystal structure (SEM-RIE) and GaAs oxide dot (AFM local oxidation) on a GaAs $105 \mathrm{~nm}$ thick slab; (b) The structure is completed by a MBE regrowth process up to $140 \mathrm{~nm}$ thickness with an embedded site-controlled InAs QD placed 20nm below the surface. AFM profiles show the change on the hole shape due to the regrowth step.

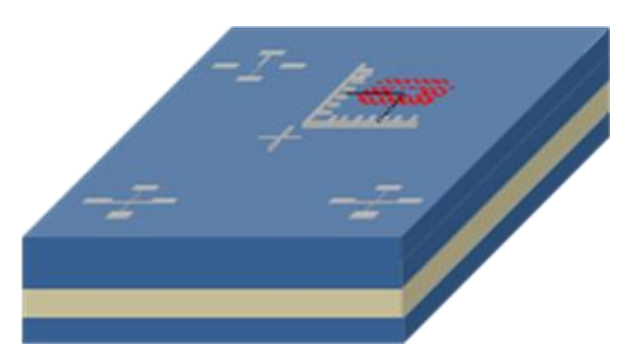

$\mathbf{a}$

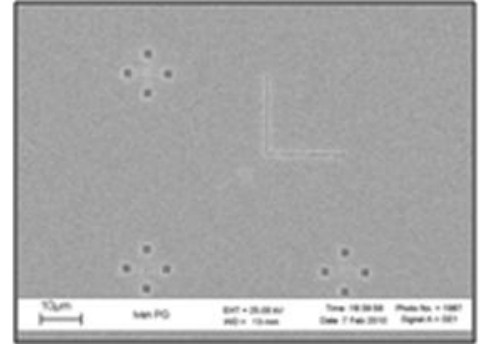

$\mathbf{b}$

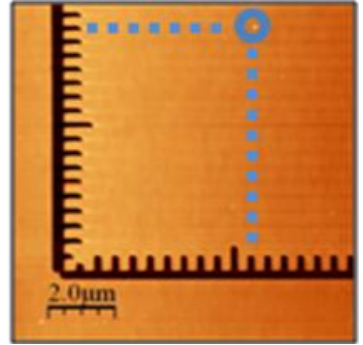

C

Figure 2. Second approximation: Deterministic cavity fabrication around site controlled InAs QD positioned respect to a prepatterned etched ruler. (a) Sketch of the final structure with a photonic cavity fabricated at the position of a site-controlled InAs QD, with coordinates set by the etched ruler. (b) SEM image of the fabricated etched ruler with the alignment marks. (c) AFM image of an actual GaAs oxide dot obtained by AFM local oxidation with known coordinates respect to the ruler. 